\title{
Molecular Simulations of Thermodynamic Properties: From Argon to Long-Chain Paraffins ${ }^{\dagger}$
}

\author{
Berend Smit \\ Shell Research B.V., Koninklijke/Shell-Laboratorium, Amsterdam, P.O. Box 38000, \\ 1030 BN Amsterdam, The Netherlands
}

\begin{abstract}
Recent progress in the simulation of complex fluids is discussed. It is shown that the use of recently developed Monte Carlo techniques allows the simulation of systems that several years ago were considered impossible to study via molecular simulations only. This development is important as it is now possible to apply simulation techniques that used to be limited to systems containing small molecules to systems which are of importance for petrochemical applications. In this short paper, this development is illustrated by two examples of practical importance: the adsorption of alkanes in zeolites and the phase behavior of long-chain alkanes.
\end{abstract}

\section{Introduction}

Most applications of molecular simulations that utilize molecular dynamics or the Monte Carlo technique are to systems containing atoms or small molecules. The reason for this is not only that ample experimental data exist for these "ideal" systems but also because the available simulation techniques are very efficient for such systems. For example, a molecular dynamics simulation of, say, a hundred methane molecules for several picoseconds is sufficient to determine most of the thermodynamic and transport properties reliably. This is in sharp contrast to a simulation of a protein or of a polymer which may take more than several nanoseconds solely to equilibrate.

From an industrial point of view, most applications of interest involve complex molecules. In the petrochemical industry long-chain alkanes are of particular interest. For this type of molecule the conventional techniques are not sufficiently efficient. Before molecular simulations can be used routinely for such systems, novel techniques that permit novel efficient simulations of these complex molecules are required.

In this short review, some recently developed Monte Carlo techniques are discussed. The methods have been developed to perform very efficient simulations of longchain alkanes. It is argued that the development of these techniques may allow simulations to become an "engineering tool" for estimating properties of systems of industrial importance.

\section{Molecular Simulation Techniques}

Before describing these new Monte Carlo methods, it is instructive to consider the limitation of the conventional simulation techniques in some detail. Let us take as an example a topic of industrial importance: the adsorption of alkanes in zeolites.

Zeolites are porous materials and are used as catalysts in petrochemical applications. A prerequisite for an understanding of the catalytic activity of these zeolites is a knowledge of the behavior of the molecules which are adsorbed in their pores. Since this type of information is extremely difficult to obtain experimentally, simulations are an attractive alternative (Thomas

\footnotetext{
${ }^{\dagger}$ Invited paper presented at the AIChE symposium on Computational Chemistry and Its Industrial Applications; AIChE Annual Meeting, San Francisco, Nov 13-18, 1994.

‡ E-mail: SMIT1@KSLA.NL.
}

(1992)). Indeed, over the last decade many simulation studies on the behavior of molecules in zeolites have been published (for a review see, for example, Catlow (1992)). A literature search on the systems that have been studied by simulations reveals that most of these studies concern the adsorption of noble gases or methane. Only a few studies of ethane or propane have been published. These simulations give important fundamental insights into the behavior of these molecules; however, from an industrial catalytic point of view the main interest is long-chain alkanes. The reason why only small molecules have been studied becomes clear from the work of June et al. (1992), in which molecular dynamics was used to investigate the diffusion of butane and hexane in the zeolite silicalite. June et al. showed that the diffusion of butane from one channel of the zeolite into another channel is very slow compared to diffusion of bulk butane. Consequently, many hours of supercomputer time were required to obtain reliable results. In addition, since the diffusion coefficient decreases significantly with increasing chain length, extrapolation of the results of June et al. suggests that many years of supercomputer time would be required to obtain comparable results for the longer alkanes.

The above example illustrates the fundamental problem of molecular dynamics, where in a simulation the approach is to mimic the behavior of the molecules as accurately as possible. If successful, all properties will be like in nature, including the diffusion. If the molecules diffuse slowly, this will be reflected in very long simulation times, and in the case of long-chain alkanes, these simulation times were much longer than we can currently afford. In principle, one can circumvent this intrinsically slow dynamics by using a Monte Carlo technique. In a Monte Carlo simulation one does not have to follow the "naturel path" and one can, for example, perform a move in which it is attempted to displace a molecule to a random position in the zeolite. If such a move is accepted, it corresponds to a very large jump in phase space.

Again, utilization of such "unnatural" Monte Carlo moves turned out to be limited to small molecules. For example, Goodbody et al. (1991) have used such Monte Carlo moves to determine the adsorption isotherm of methane in silicalite. In such a simulation one can observe that out of the 1000 attempts to move a methane molecule to a random position in the zeolite, 999 attempts will be rejected because the methane molecule overlaps with a zeolite atom. If we were to 


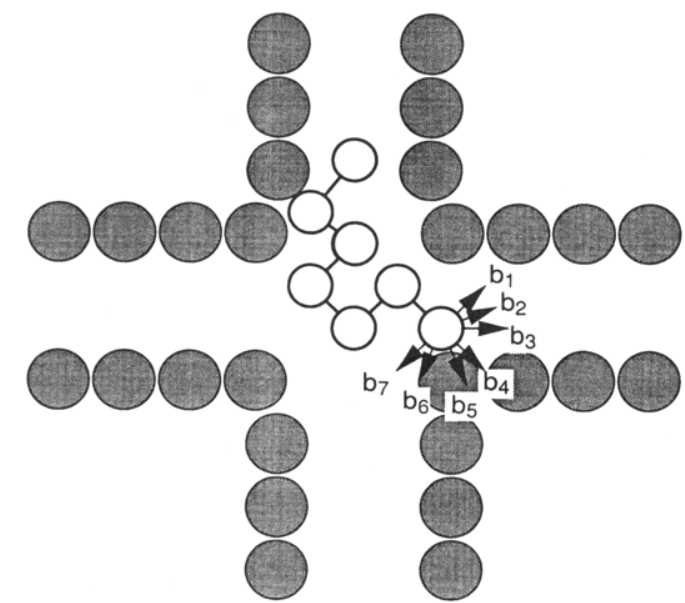

Figure 1. Schematic drawing of the growing of an alkane in a zeolite in a configurational-bias Monte Carlo move. The shaded circles represent the atoms of the zeolite and the open circles those of the alkane. The set of $k$ trial orientations $\left\{\mathbf{b}_{1}, \mathbf{b}_{2}, \ldots, \mathbf{b}_{k}\right\}$ are indicated by arrows.

perform a similar move with an ethane molecule, we would need on the order of $1000 \times 1000$ attempts to have one that was successful. Clearly, this random insertion scheme will break down for any but the smallest alkanes.

To make Monte Carlo moves of long-chain molecules possible, Siepmann and Frenkel (1992) have developed the configurational-bias Monte Carlo technique for lattice models. This technique is based on the early work of Rosenbluth and Rosenbluth (1955) and Harris and Rice (1988). This technique has since been extended to off-lattice systems by Frenkel et al. (1992) and de Pablo et al. (1992).

The principle idea behind the configurational-bias Monte Carlo technique is to grow a molecule atom by atom instead of attempting to insert the entire molecule at random. Figure 1 shows one of the steps in this algorithm. Assume that $i-1$ atoms have been grown and that we now attempt to insert atom $i$. We generate a set of $k$ trial positions, denoted by the arrows $\mathbf{b}_{1}, \ldots$, $\mathbf{b}_{k}$. Out of these we select the one with the lowest energy with the highest probability; i.e., position $i$ is selected with a probability

$$
P(i)=\frac{\exp \left[-\beta u\left(\mathbf{b}_{i}\right)\right]}{\sum_{j=1}^{k} \exp \left[-\beta u\left(\mathbf{b}_{j}\right)\right]}
$$

where $u$ denotes the energy of an atom. This equation ensures that conformations with a low energy have a high probability of being selected. The above procedure is repeated until the entire molecule has been grown. It is important to note that this growing procedure introduces a bias, such that only the most favorable configurations will be generated. If one were to use the ordinary Metropolis acceptance rule, such a bias in the configurations of the molecules would lead to an incorrect distribution of configurations. Frenkel et al. (1992) have shown that this bias can be corrected by adjusting the acceptance rules.

It is not my purpose here to give an extensive discussion on the implementation of this algorithm as details can be found in Frenkel et al. (1992), de Pablo et al. (1992), Siepmann and McDonald (1993), Siepmann (1993), and Smit and Siepmann (1994a). It is, however,

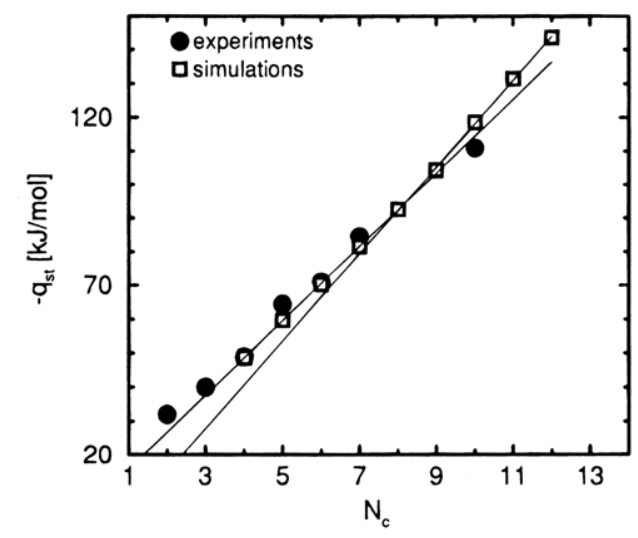

Figure 2. Heats of adsorption of the alkanes in silicalite. The solid lines are linear fits to the data, which give a slope of $11 \mathrm{~kJ} /$ mol per carbon atom for $\mathrm{C}_{4}$ to $\mathrm{C}_{8}$ and $13 \mathrm{~kJ} / \mathrm{mol}$ per carbon atom for $\mathrm{C}_{8}$ to $\mathrm{C}_{12}$. The experimental data (denoted by $\bullet$ ) are compiled from Abdul-Rehman et al. (1990), Dubinin et al. (1989), Hufton and Danner (1993), Stach et al. (1984), Stach et al. (1986), and Thamm (1987).

instructive to discuss some of the advantages of this novel technique. First of all, it is important to note that this is a Monte Carlo technique and therefore cannot be used directly to determine transport properties. One may consider using the configurational-bias Monte Carlo technique to enhance the equilibration of the system, but to determine the transport properties, one has to do a molecular dynamics simulation. In many cases the interest is primarily in the thermodynamic properties, in which case the configurational-bias Monte Carlo technique can be extremely useful since it can be 100 orders of magnitude (!) more efficient than the conventional techniques. In the next section, we demonstrate that this enormous increase in efficiency allows us to perform simulations that were considered impossible only a few years ago.

\section{Applications}

In the Introduction we used the adsorption of molecules as an example to illustrate the type of problem one can encounter in simulating systems that exhibit slow diffusion. Smit and Siepmann (1994a,b) have used the configurational-bias Monte Carlo technique to study the energetics and siting of alkanes in the zeolites silicalite and mordenite. Figure 2 shows that the simulations predict heats of adsorption of the longer chain alkanes in silicalite that are in good agreement with the experimental data. It is interesting to note that the heats of adsorption show an unexpected chain length dependence. The heat of adsorption increases $11 \mathrm{~kJ} / \mathrm{mol}$ per $\mathrm{CH}_{2}$ group for the short-chain alkanes, while for the long-chain alkanes a value of $13 \mathrm{~kJ} / \mathrm{mol}$ per $\mathrm{CH}_{2}$ group has been obtained. Smit and Siepmann attributed this chain length dependence to a preferential siting of the long-chain alkanes in the linear channels of silicalite. This type of information on the behavior of the adsorbed molecules at a molecular level turned out to be extremely difficult to deduce from experimental data.

Simulations can also be used to determine adsorption isotherms. Adsorption isotherms are of practical importance as they give information on the number of molecules adsorbed in the pores of a zeolite as a function of the pressure of the reservoir. Adsorption isotherms are conveniently determined from simulations in the 


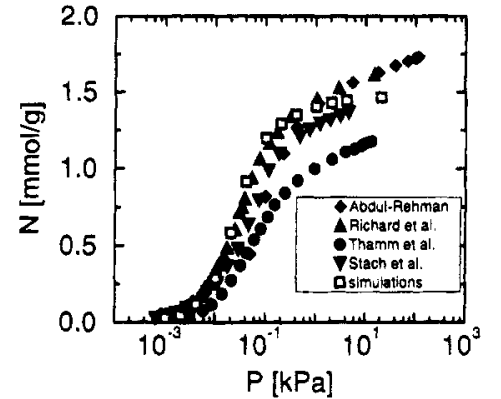

Figure 3. Adsorption isotherms of butane. The closed symbols are experimental data [Abdul-Rehman et al. (1990), Richard and Rees (1987), Stach et al. (1986), Thamm (1982)] and the open symbols the results from simulations at $T=298 \mathrm{~K}$.

grand-canonical ensemble. In this ensemble the temperature and chemical potential are imposed, but the number of particles is allowed to fluctuate. In Figure 3 the experimental adsorption isotherms of butane in silicalite are compared with the results from simulations. The scatter in the experimental data illustrates that measurement of adsorption isotherms on welldefined silicalite samples is very difficult. The simulation data are in very good agreement with the experimental data.

Another problem of practical importance for the petrochemical industry is the phase behavior of longchain alkanes. Alkanes are thermally unstable above approximately $650 \mathrm{~K}$, which makes experimental determination of the critical point of alkanes which are longer than decane extremely difficult. The longer alkanes, however, are present in mixtures. In these mixtures, the number of components can be so large that it is not practical to determine all phase diagrams experimentally. One therefore has to rely on predictions made by equations of state. The parameters of these equations of state are directly related to the critical properties of the pure components. Therefore, the critical properties of the long-chain alkanes are of importance in the design of petrochemical processes, even if they are unstable close to the critical point. Unfortunately, experimental data are scarce and contradictory, and one has to rely on semiempirical methods to estimate the critical properties [Tsonopoulos (1987)]. Siepmann et al. (1993) and Smit et al. (1995) have used a combination of the Gibbs ensemble and configurational-bias Monte Carlo technique to simulate the phase behavior of the longer alkanes at conditions where experiments are not (yet) feasible.

In the Gibbs-ensemble scheme, simulations of the liquid and vapour phases are carried out in parallel [Panagiotopoulos (1987), Panagiotopoulos et al. (1988), Smit et al. (1989)]. Monte Carlo rules, which allow for changes in the number of particles and the volume, ensure that the two boxes are in thermodynamic equilibrium. Since the two boxes are not in "physical contact", there is no interface and the bulk properties of the two coexisting phases can be obtained directly with a surprisingly small number of particles. This makes the Gibbs-ensemble scheme extremely efficient for phase equilibrium calculations. The major limitation of the Gibbs-ensemble technique used to be that one of the steps involves the exchange of particles between the two boxes. For liquids consisting of small molecules this does not cause serious problems. However, for chain molecules the probability of successful exchanges can become very small for similar reasons as discussed above for the adsorption of alkanes in zeolites. This

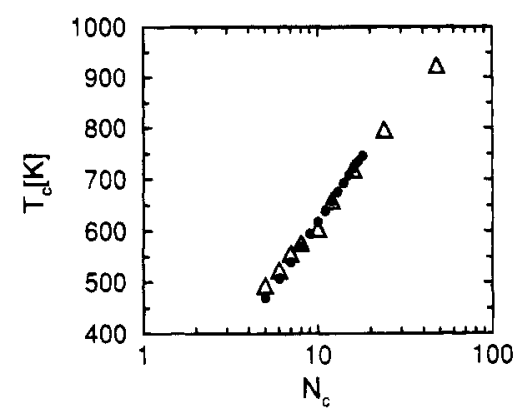

Figure 4. Critical temperature $T_{\mathrm{c}}$ as a function of carbon number $N_{c}$. $\Delta$ represents the simulation data and the experimental data from Anselme et al. (1990) and Tsonopoulos and Tan (1993).

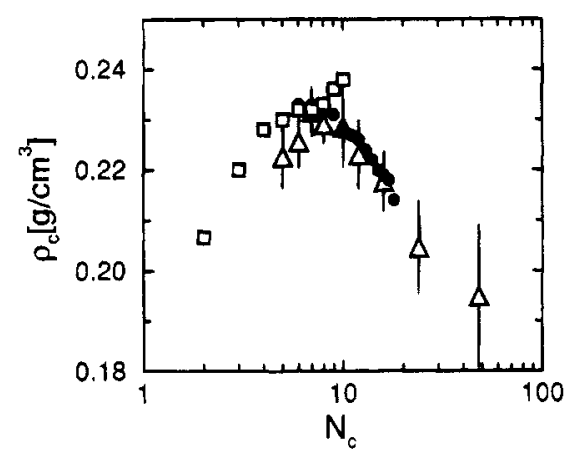

Figure 5. Critical density $\rho_{\mathrm{c}}$ as a function of carbon number $N_{c}$. $\Delta$ represents the simulation data, the experimental data from Anselme et al. (1990), and $\square$ the data of Steele (as published in Tsonopoulos and Tan (1993)).

problem can be resolved by using the configurationalbias Monte Carlo method [Mooij et al. (1992), Laso et al. (1992)], the details of which are described by Smit et al. (1995).

Figure 4 is a plot of the critical temperature versus carbon number. The simulations reproduce the experimental critical temperatures very well. There is, however, considerable disagreement between the various experimental estimates of the critical densities. Much of our current knowledge of the critical properties of the higher alkanes is based on extrapolations of fits of the experimental data up to $\mathrm{C}_{8}$. The most widely used extrapolations assume that the critical density is a monotonically increasing function of the carbon number, approaching a limiting value for the very long alkanes [Tsonopoulos (1987), Tsonopoulos and Tan (1993)]. In contrast to these predictions based on extrapolations, the recent experimental data of Anselme et al. (1990) indicate that the critical density has a maximum for $\mathrm{C}_{8}$ and then decreases monotonically. The data of Steele (as reported in Tsonopoulos and Tan (1993)), however, do not give any evidence for such a maximum (see Figure 5). The simulations of Siepmann et al. indicate the same trend as that observed by Anselme et al. Since these calculations can also be performed for much longer alkanes, the results of Siepmann et al. strongly support those of Anselme et al.

\section{Concluding Remarks}

Industrial applications of molecular simulations to determine thermodynamic properties usually involve systems containing complex fluids. For this type of system, in particular for a system containing long-chain alkanes, the conventional simulation techniques are not sufficiently efficient. It has been shown that the recently developed configurational-bias Monte Carlo 
technique is particularly suited to simulate the thermodynamic properties of these complex fluids.

The use of the novel techniques is illustrated with two examples: the simulation of the adsorption of long-chain alkanes in zeolites and the phase behavior of long-chain alkanes at high temperatures. These examples demonstrate that simulations provide data under conditions where direct experiments are not currently feasible.

\section{Literature Cited}

Abdul-Rehman, H. B.; Hasanain, M. A.; Loughlin, K. F. Quaternary, ternary, binary, and pure component sorption on zeolites. 1. Light alkanes on Linde S-115 silicalite at moderate to high pressures. Ind. Eng. Chem. Res. 1990, 29, 1525-1535.

Anselme, M. J.; Gude, M.; Teja, A. S. The critical temperatures and densities of the n-alkanes from pentane to octadecane. Fluid Phase Equilib. 1980, 57, 317-326.

Catlow, C. R. A., Ed. Modelling of Structure and Reactivity in Zeolites; Academic Press: London, 1992.

de Pablo, J. J.; Laso, M.; Suter, U. W. Simulation of polyethylene above and below the melting point. J. Chem. Phys. 1992, 96 , 2395-2403.

Dubinin, M. M.; Rakhmatkariev, G. U.; Isirikyan, A. A. Adsorption energetics of hydrocarbons on silicalite. Izv. Akad. Nauk SSSR, Ser. Khim. 1989, 10, 2333-2335.

Frenkel, D.; Mooij, G. C. A. M.; Smit, B. Novel scheme to study structural and thermal properties of continuously deformable molecules. J. Phys.: Condens. Matter 1992, 4, 3053-3076.

Goodbody, S. J.; Watanabe, K.; MacGowan, D.; Walton, J. P. R. B.; Quirke, N. Molecular simulations of methane and butane in silicalite. J. Chem. Soc., Faraday Trans. 1991, 87, 19511958.

Harris, J.; Rice, S. A. A lattice model of a supported monolayer of amphiphilic molecules: Monte Carlo simulations. J. Chem. Phys. 1988, 89, 1298-1306.

Hufton, J. R.; Danner, R. P. Chromatographic study of alkanes in silicalite: equilibrium properties. AIChE J. 1993, 39, 954-961.

June, R. L.; Bell, A. T.; Theodorou, D. N. Molecular dynamics of butane and hexane in silicalite. J.Phys. Chem. 1992, 96, 10511060 .

Laso, M.; de Pablo, J. J.; Suter, U. W. Simulation of phase equilibria for chain molecules. J. Chem. Phys. 1992, 97, 28172819.

Mooij, G. C. A. M.; Frenkel, D.; Smit, B. Direct simulation of phase equilibria of chain molecules. J. Phys.: Condens. Matter 1992, 4, L255-L259.

Panagiotopoulos, A. Z. Direct determination of phase coexistence properties of fluids by Monte Carlo simulation in a new ensemble. Mol. Phys. 1987, 61, 813-826.

Panagiotopoulos, A. Z.; Quirke, N.; Stapleton, M.; Tildesley, D. J Phase equilibria by simulations in the Gibbs ensemble. Mol. Phys. 1988, 63, 527-545.

Richard, R. E.; Rees, L. V. C. Sorption and packing of n-alkane molecules in ZSM-5. Langmuir 1987, 3, 335-340.

Rosenbluth, M. N.; Rosenbluth, A. W. Monte Carlo simulations of the average extension of molecular chains. J. Chem. Phys. $1955,23,356$.
Siepmann, J. I. Configurational-bias Monte Carlo: Background and Selected Applications. In Computer simulation of biomolecular systems: theoretical and experimental applications; van Gunsteren, W. F., Weiner, P. K., Wilkinson, A. J., Eds.; ESCOM Science Publisher: Leiden, The Netherlands, 1993; pp 249-264.

Siepmann, J. I.; Frenkel, D. Configurational-bias Monte Carlo: a new sampling scheme for flexible chains. Mol. Phys. 1992, 75, $59-70$.

Siepmann, J. I.; McDonald, I. R. Monte Carlo study of the properties of self-assembled monolayers formed by the adsorption of $\mathrm{CH}_{3}\left(\mathrm{CH}_{2}\right)_{15} \mathrm{SH}$ on the (111) surface of gold. Mol. Phys. $1993,79,457-473$.

Siepmann, J. I.; Karaborni, S.; Smit, B. Simulating the critical properties of complex fluids. Nature 1993, 365, 330-332.

Smit, B.; Siepmann, J. I. Computer simulations of the energetics and siting of n-alkanes in zeolites. J. Phys. Chem. 1994a, 98, 8442-8452.

Smit, B.; Siepmann, J. I. Simulating the adsorption of alkanes in zeolites. Science 1994b, 264, 1118-1120.

Smit, B.; de Smedt, Ph.; Frenkel, D. Computer simulations in the Gibbs ensemble. Mol. Phys. 1989, 68, 931-950.

Smit, B.; Karaborni, S.; Siepmann, J. I. Computer simulations of vapour-liquid phase equilibria of n-alkanes. J. Chem. Phys. 1995, 102, 2126

Stach, H.; Thamm, H.; Jänchen, J.; Fiedler, K.; Schirmer, W. Experimental and theoretical investigation of the adsorption of n-paraffins, $n$-olefins and aromatics on silicalite. In New Developments in Zeolite Science and Technology; Olsen, D., Bisio, A., Eds.; Proceedings of the 6th International Zeolite Conference; Butterworth: Guildford, U.K., 1984; pp 225-231.

Stach, H.; Lohse, U.; Thamm, H.; Schirmer, W. Adsorption equilibria of hydrocarbons on highly dealuminated zeolites. Zeolites 1986, 6, 74-90.

Thamm, H. Adsorption site heterogeneity in silicalite: a calorimetric study. Zeolites 1987, 7, 341-346.

Thamm, H.; Stach, H.; Schirmer, W.; Fahlke, B. Charakterisierung von mikroporösen $\mathrm{SiO}_{2}$ and zeolithischen Molekularsieben durch Kalorimetriche Bestimmung der Adsorptionswechselwirkung mit n-Butan. Z. Phys. Chem., Leipzig 1982, 263, 461469.

Thomas, J. M. Solid acid catalysts. Sci. Am. 1992, 266, 82-88.

Tsonopoulos, C. Critical constant of normal alkanes from methane to polyethylene. AIChE J. 1987, 33, 2080-2083.

Tsonopoulos, C.; Tan, Z. Critical constant of normal alkanes from methane to polyethylene. II. Application of the Flory theory. Fluid Phase Equilib. 1993, 83, 127-138.

Received for review April 3, 1995

Revised manuscript received July 12, 1995 Accepted July $24,1995^{\circledR}$

IE9502260

${ }^{\otimes}$ Abstract published in Advance ACS Abstracts, November $1,1995$. 